Regular Article

\title{
Wetting dynamics of polydimethylsiloxane mixtures on a poly(ethylene terephthalate) fiber
}

\author{
Yichuan Zhang a,b,*, Arnaud Vandaele ${ }^{\mathrm{b}}$, David Seveno ${ }^{\mathrm{a}}$, Joël De Coninck ${ }^{\mathrm{b}}$ \\ a Department of Materials Engineering, KU Leuven, 3001 Leuven, Belgium \\ ${ }^{\mathrm{b}}$ Laboratory of Surface and Interfacial Physics, Université de Mons, 7000 Mons, Belgium
}

\section{A R T I C L E I N F O}

\section{Article history:}

Received 26 March 2018

Revised 22 April 2018

Accepted 23 April 2018

Available online 24 April 2018

\section{Keywords:}

Dynamic contact angle

Capillary rise

Molecular-kinetic theory

Hydrodynamic approach

\begin{abstract}
A B S T R A C T
Hypothesis: The wetting dynamics of liquids with identical surface tensions are mostly controlled by their viscosities. We therefore hypothesized that the wetting dynamics of one-(pure liquid) and two-component (mixture) polydimethylsiloxane (PDMS) on a poly(ethylene terephthalate) (PET) fiber with similar surface tensions and viscosities should be controlled by the same underlying physical mechanisms.

Experiments: We studied the capillary rise of PDMS liquids on a PET fiber. We compared the different contact angle relaxations and characterized the transitions between the molecular-kinetic theory (MKT) and hydrodynamic approach (HD) for the PDMS mixtures and the pure liquids as a function of their viscosities. Findings: Compared to the pure PDMS liquid with a viscosity of $20 \mathrm{~mm}^{2} / \mathrm{s}$ that presents a contact angle relaxation following a $t^{-1 / 2}$ scale law in agreement with HD, the PDMS mixture with a higher viscosity $\left(27.4 \mathrm{~mm}^{2} / \mathrm{s}\right)$ shows a $t^{-1}$ behavior predicted by the MKT. Moreover, the transition between MKT and HD appears in a regime with higher viscosities for PDMS mixtures than for pure liquids. Surface segregation of shorter PDMS chains or precursor film may be responsible for this shift.
\end{abstract}

(c) 2018 Elsevier Inc. All rights reserved.

\section{Introduction}

Wetting dynamics is of fundamental importance in numerous processes, such as adhesion, secondary oil recovery, painting,

* Corresponding author at: Department of Materials Engineering, KU Leuven, 3001 Leuven, Belgium, and Laboratory of Surface and Interfacial Physics, Université de Mons, 7000 Mons, Belgium.

E-mail address: yichuan.zhang@kuleuven.be (Y. Zhang). liquid-based microfluidic systems, where capillary forces predominate or affect their efficiency and stability. As a consequence, wetting phenomena have been studied extensively, from both numerical and experimental aspects [1-3]. Among them, the understanding of a droplet spreading on a flat substrate is relatively well documented. However, the wetting behavior of a fiber partially immersed in a liquid bath is significantly different from that of a droplet spreading on a flat substrate due to the geometry of the fiber. For example, a fluid that can form a wetting film on a 
smooth substrate may not wet the fiber made of the same material in a similar way [4]. In fact, these different wetting behaviors stem mainly from the opposite sign of the two principal radii of curvature around the fiber $[5,6]$. This has been recognized when gravity is negligible. In the case of droplet spreading on a flat substrate, the radii of curvature become greater till equilibrium, reaching possibly an infinite value in the extreme case of the formation of a wetting film. This fundamental difference between the two geometries indicates that the study of the liquid-fiber system should exhibit distinctive wetting characteristics.

To describe the dynamics of wetting, two main theoretical approaches have been proposed: the hydrodynamic approach (HD) and the molecular-kinetic theory (MKT), which differ from each other mostly in the consideration of the channel of dissipation $[2,3,7,8]$. The first approach emphasizes the dissipation due to viscous bending generated during displacement of the contact line [8-11], and the MKT proposed by Blake and Hayes concentrates on the frictional processes occurring in the vicinity of the advancing contact line [12-16]. Since these models have already been extensively described in the literature, we will not repeat them here. When applied to the spreading of liquid droplets on a flat surface with vanishing advancing contact angle $\left(\theta_{t}\right)$, the two models predict slightly different power laws. Typically, $\theta_{t}$ varies as $t^{-3 / 10}$ and $t^{-3 / 7}$ for the HD and MKT, respectively $[10,14,17,18]$. It implies that the difference between these two regimes is not experimentally easily distinguishable on flat surfaces. For the case of fibers, Quéré et al. $[19,20]$ suggested that the capillary rise along a thin vertical fiber can be characterized by $\theta_{t} \sim t^{-1 / 2}$ in agreement with the HD approach, whereas Seveno et al. [21] predicted that $\theta_{t} \sim$ $t^{-1}$ when the contact-line dynamics follows the MKT model and this result has been validated by large scale molecular dynamics simulations. Besides, Vega et al. [22] have studied experimentally the capillary rise of viscous liquids round a poly(ethylene terephthalate) (PET) fiber and observed that $\theta_{t} \sim t^{-1}$ for low-viscosity liquids (in agreement with MKT) and $\theta_{t} \sim t^{-1 / 2}$ for high-viscosity liquids (in agreement with HD). These works suggest that, compared to flat geometry, it presents some advantages to use cylindrical fibers when the dissipation regimes have to be unambiguously distinguished.

Just as blending bulk polymers to obtain a desired physical property [23], addition of a second component to a liquid can help to tailor wetting properties $[24,25]$. Compared to one-component systems, the number of works related to two-component ones is rather limited despite their adjustability and versatility [26-28]. The root of this issue might be due to the fact that one faces some tough issues in this endeavor. For example, the mutual compatibility of two components has to be taken into account [29]. Besides, the simultaneous variation of surface tension and viscosity makes it difficult to distinguish the roles of these two factors for processes driven by wetting [30]. This indicates that the problem requires some clarification.

In the present work, we report on the spreading dynamics, from an infinite reservoir, of one- and two-component polymer liquids around a PET fiber. The primary aim is to explore the possible existence of the different dissipation mechanisms that may appear in two-component systems, and to provide insight into the role of short polymer chains in the wetting process. Two types of polydimethylsiloxane (PDMS) with viscosities of $5 \mathrm{~mm}^{2} / \mathrm{s}$ (PDMS5) and $500 \mathrm{~mm}^{2} / \mathrm{s}$ (PDMS500) were selected. First, according to the work of Vega et al. [22], PDMS500 and PDMS5 respectively follow the HD model and the MKT. Besides, the solubility parameter, $\sim 14.9 \mathrm{MPa}^{1 / 2}$ [31,32], of PDMS liquids is nearly independent on viscosity or molecular weight, so that a mixture of these two liquids is thermodynamically compatible according to the Hildebrand-Scatchard equation [33]. Their compatibility is also supported by the experimental work of Fondecave and Brochard-
Wyart [34]. Additionally, the viscosity of the two-component PDMS systems can be tailored by their ratio, simultaneously maintaining a nearly constant surface tension. These characteristics offer a way to study the dissipation mechanisms of the liquids with various viscosities, and also open the door to a better understanding of the role of short polymer chains in two-component polymer systems.

\section{Materials and methods}

\subsection{Materials}

The fiber is a PET monofilament fiber with $0.8 \mathrm{~mm}$ diameter from Goodfellow. The polymer liquids are linear PDMS provided by Sigma-Aldrich Chemical Co. and used as received. The PDMS5 liquid, with short chains, has a number-average molecular weight of around $800 \mathrm{~g} / \mathrm{mol}$, and its counterpart, PDMS500, with long chains has a number-average molecular weight of around 17,300 $\mathrm{g} / \mathrm{mol}$. Both molecular weights are below the critical molecular weight for entanglement ( $\mathrm{Mc} \approx 29,000 \mathrm{~g} / \mathrm{mol}$ ) [31], for which reptation is expected [35]. Therefore, the polymer chains of the PDMS5 and PDMS500 liquids can be represented by Rouse chains without consideration of entanglement as well as disentanglement when short and long chains are combined.

The 2-component PDMS mixtures were prepared by magnetic stirring for more than $16 \mathrm{~h}$ with mass ratio (PDMS5/PDMS500) of $75 \% / 25 \%, 70 \% / 30 \%, 65 \% / 35 \%, 60 \% / 40 \%, 55 \% / 45 \%, 50 \% / 50 \%$ and $25 \% / 75 \%$. For the sake of brevity, the solutions with those ratios are referred to $75 / 25,70 / 30,65 / 35,60 / 40,55 / 45,50 / 50$ and $25 / 75$ liquids. The mixtures were always used just prior to their mixing preparations, so these liquids were assumed to be homogenous. Surface tensions were measured by the pendant drop method using a Krüss drop-shape analyzer (DSA100) at $20^{\circ} \mathrm{C}$. Dynamic viscosities of all liquids, including the pure PDMS5 and PDMS500, were measured with a Brookfield DV-II + PRO digital viscometer at the same temperature. Kinematic viscosity (the ratio of the dynamic viscosity to the density of liquid) is also presented for comparison with the work of Vega et al. [22]. The surface tension and viscosity values are shown in Fig. 1. It indicates that surface tension values are nearly constant while the viscosity values increase dramatically with addition of PDMS500. Table S1 (Supplementary material) lists the capillary length of the liquids ( $a=\sqrt{\gamma / \rho g}$, with $\gamma$ the liquid-vapor surface tension, $\rho$ the liquid density, and $g$ the gravitational acceleration), the Bond number

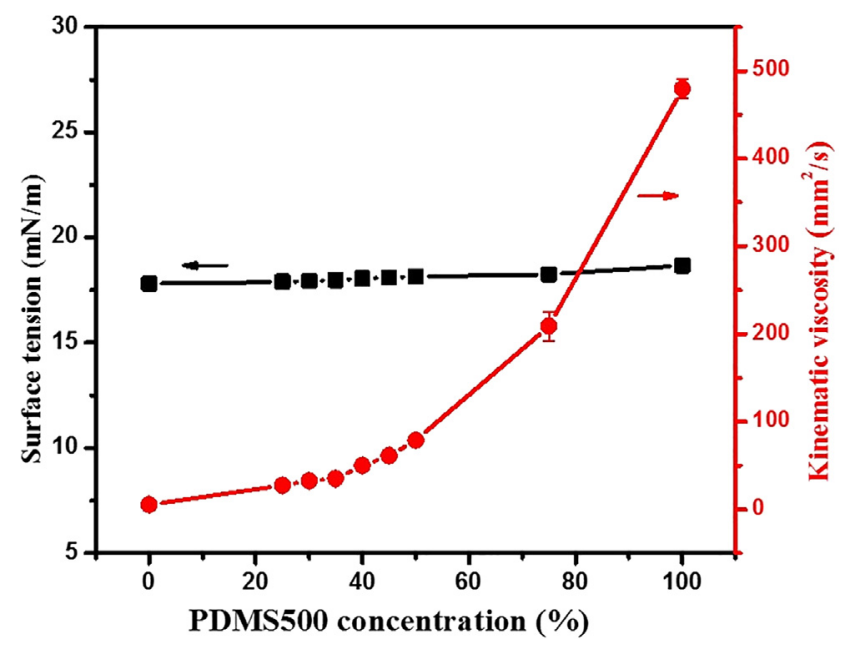

Fig. 1. Surface tensions and viscosities of the one- and two-component PDMS liquids versus PDMS500 concentration. 
( $B o=r_{0} / a$ with $r_{0}$ the fiber radius) and the capillary number ( $C a=\eta V / \gamma$, with $V$ the contact line velocity and $\eta$ the dynamic viscosity). The Bond number permits to distinguish the planar from the fiber case [20]. If $B o$ is larger than one unity, then the fiber can be treated as a planar surface. Here Bo (in Table S1) is almost constant and equal to $0.27 \sim 0.28$, which means that the filament must be treated as a cylinder. The capillary number does not exceed 0.03 in the present experiments. In such conditions, the liquid/vapor interface has a quasi-static profile and obeys the Laplace's equation.

\subsection{Cleaning of the PET fibers}

Prior to the wetting experiments, all possible organic contaminations are first pulled off by rubbing the fiber with a soap (RBS-35 from Chemical Products). The fibers are then sonicated for $30 \mathrm{~min}$ in an ultrasound bath of pure water (Milli-Q water) and finally in another ultrasound bath of pure 2-propanol (J. T. Baker with a purity of $99.5 \%$ ) for $1 \mathrm{~h}$. The cleaned fiber is then dried under a flux of nitrogen. The fibers are always cleaned just before implementing the experiments.

\subsection{Dynamic wetting measurements}

The experimental setup is sketched in Fig. 2. The fiber is attached to a sample holder of a dip coater setup allowing the fiber to move down into the liquid reservoir. A grid line is used to adjust the verticality of the fiber. The downward motion of the sample is operated at a velocity of $5 \mathrm{~mm} / \mathrm{min}$ and is stopped immediately when the fiber contacts the liquid. This is carefully checked by analyzing the successive images just before and after the meniscus rise starts. The meniscus profile is captured using a high speed camera (C-MOS camera, Vosskühler HCC-1000) with an additional LED backlight. The spreading experiments were repeated at least three times for each PDMS liquid. A good repeatability is evidenced by standard deviation with values lower than $3^{\circ}$.
Images of the meniscus were analyzed (around typically 500 images per experiment) using G-fiber [22] to obtain values of the contact angle and meniscus height simultaneously. Fig. 3 presents a typical example of a contact angle measurement. It follows two steps: first, the fiber/air and liquid/air interfaces are detected on both sides of the fiber; subsequently, the shape of the liquid/air interface is adjusted by a solution of Laplace's equation to calculate the contact angle and the meniscus height. However, it is difficult to measure small contact angles, typically below $15^{\circ}$, by this optical method due to the high curvature of the liquid/vapor interface close to the fiber surface. Therefore, the relaxation of small contact angles is not studied here.

\subsection{Theoretical considerations}

It has been widely accepted that both dissipations in HD and the frictional processes in MKT do exist simultaneously, and several attempts to work out a combined model have been proposed [14]. The extended De Ruijter combined model [36] for fiber geometry incorporating HD and MKT models is employed to distinguish the dominant channels of dissipation for the PDMS liquids. The relationship between dynamic contact angle $\theta_{t}$, dynamic meniscus height $y_{t}$ and the contact line velocity $V$ is given by

$V=\frac{d y_{t}}{d t}=\frac{\gamma\left(\cos \theta_{0}-\cos \theta_{t}\right)}{\zeta+\frac{6 \eta}{\theta_{t}} \ln \left(\frac{x_{\max }}{x_{\min }}\right)}$

where $\theta_{0}$ is equilibrium contact angle, $\zeta$ is the friction associated with the dissipation channel occurring in the vicinity of the contact line, $x_{\max }$ is on the order of millimeters and $x_{\min }$ is of molecular size [37]. Assumed that values of $\theta_{t}$ are small and $\theta_{0}=0^{\circ}$, we can obtain

$$
\frac{d \theta_{t}}{d t} \approx-\frac{\gamma\left(\frac{\theta_{t}{ }^{2}}{2 r_{0}}\right)}{\zeta+\frac{6 \eta \ln \left(\frac{x_{\max }}{x_{\min }}\right)}{\theta_{t}}}
$$

Whenever there is no friction at the contact line, it leads to

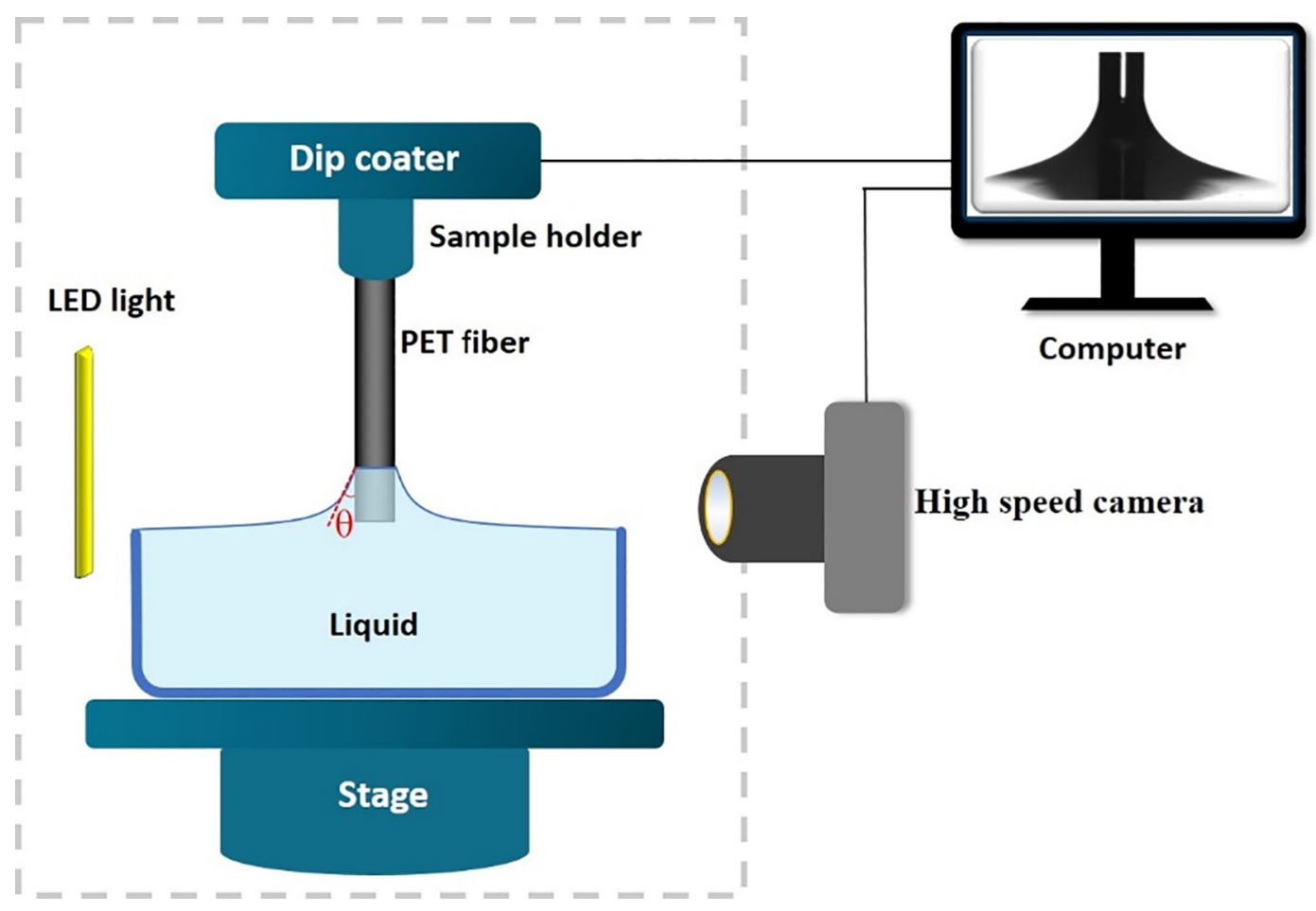

Fig. 2. Schematic of the experimental setup. 


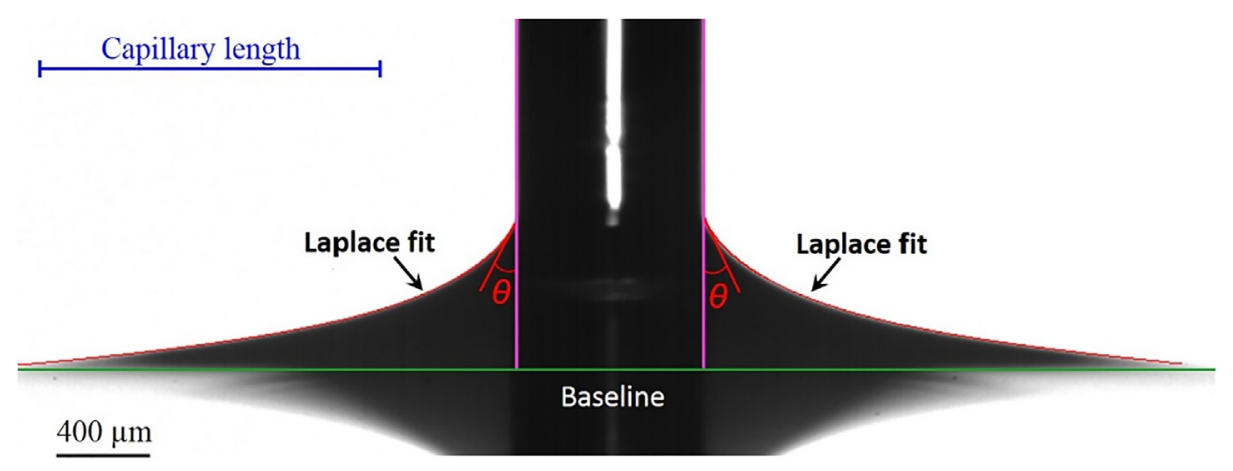

Fig. 3. A typical example of a contact angle measurement. The best Laplace’s fit of the liquid/air interface gives $\theta=25.1 \pm 0.4$.

$\theta_{t} \propto t^{-1 / 2}$

and for the other case with a small viscosity, it gives

$\theta_{t} \propto t^{-1}$

The detailed theoretical derivations of Eqs. (3) and (4) can be found in our previous works $[21,22]$, which were validated numerically and experimentally [19-22].

\section{Results and discussion}

\subsection{Contact angle relaxation and meniscus height dynamics}

Fig. $4 \mathrm{a}$ and $\mathrm{b}$ show the contact angle relaxation and meniscus height dynamics for the liquids. The curves of the 65/35 and $60 / 40$ liquids lie between the ones of the $70 / 30$ and 55/45 liquids, and are not shown for clarity. The process of capillary rise completes in a few seconds, therefore the possible effect of the evaporation of liquids is not considered here. It is expected that the relaxation of the contact angles and meniscus height dynamics slow down for liquids with a higher concentration of PDMS500 due to a rapid increment of their viscosities. It should be noted that when the fiber is in contact with the liquid, the meniscus starts to rise spontaneously and instantaneously around the fiber. The rela-

(a)

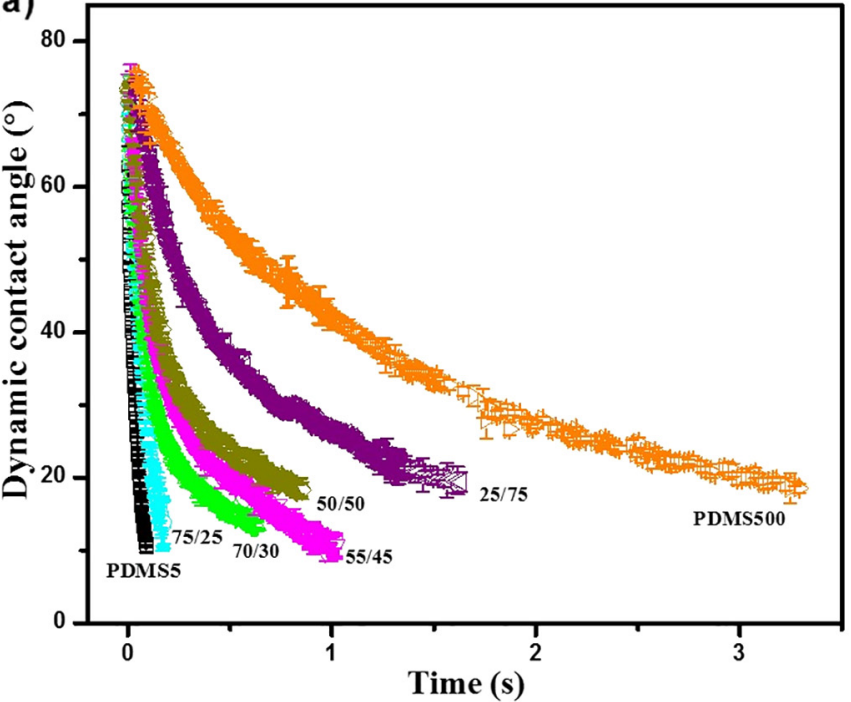

tively low surface tension of these liquids (Fig. 1) compared to the critical surface tension of the PET fiber $\left(=42 \mathrm{mN} \cdot \mathrm{m}^{-1}\right)$ ensure that there is no significant additional elastic stress during the breakup of the liquid/air interface.

Fig. 5 shows the same contact angle dynamics for $\theta_{t} \leq 45^{\circ}$ on logarithmic scales. As clearly seen, it presents two different behaviors when the meniscus approaches equilibrium. The PDMS5 and $75 / 25$ liquids show a slope of around -1 , whereas the others present a slope of around -0.5 . Their values and deviations are summarized in Table 1. Apparently, the contact angle dynamics of the PDMS5 and 75/25 liquids follow Eq. (4) and the behaviors of the other liquids can be described by Eq. (3). The dynamic contact angles are assessed below $45^{\circ}$ as the trigonometric functions can then be approximated to their Taylor expansions as required by the derivation of the scaling laws in Eq. (2). Independent tensiometric measurements also confirm that $\theta_{0}$ is equal to $0^{\circ}$ (See Fig. S1 in Supplementary material).

It should be noted that the early stage of the wetting dynamics of low viscous liquids can be affected by inertia and its effect should be eliminated. Clanet and Quéré [20] suggested that the meniscus rise on a small fiber driven by inertia can be described by

$y_{t} \approx \alpha\left(\frac{\gamma r_{0} t^{2}}{\rho}\right)^{1 / 4}$

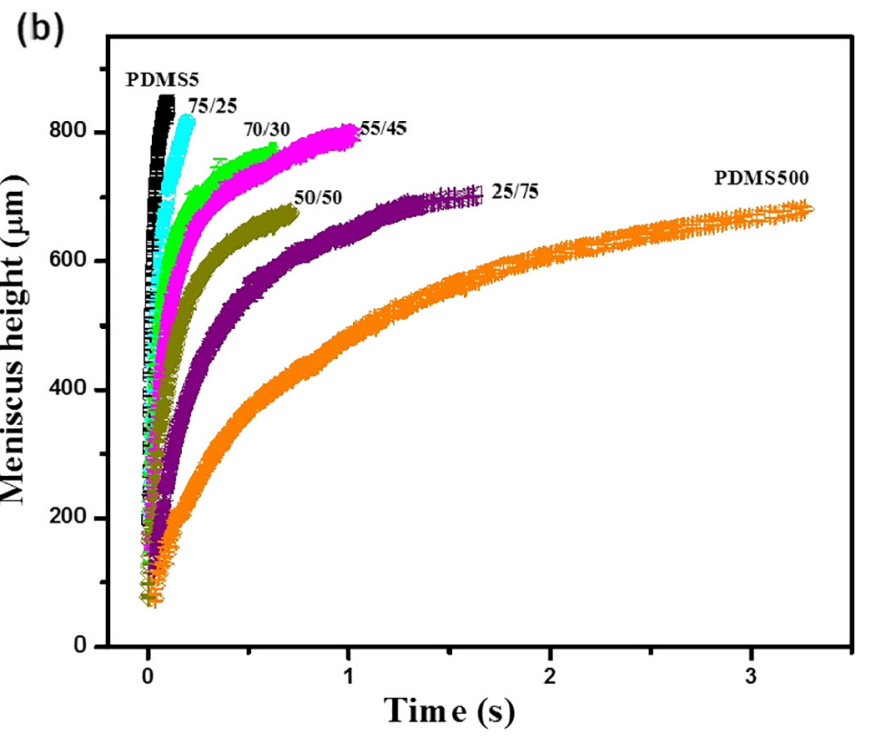

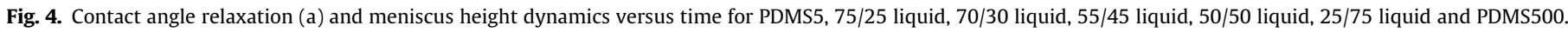
The curves 65/35 and 60/40 liquids are not shown for clarity. 


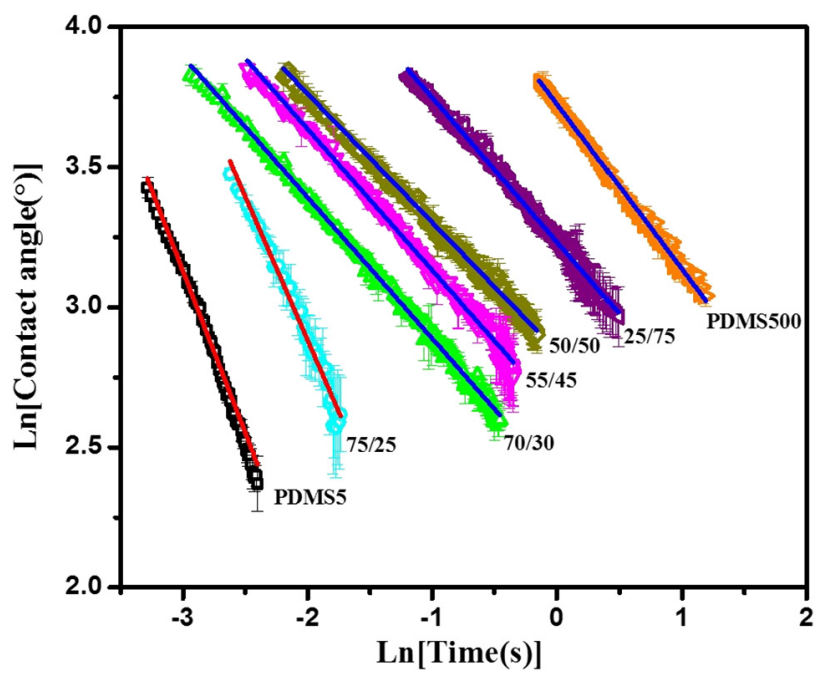

Fig. 5. Late stage contact angle dynamics vs time for PDMS5, 75/25 liquid, 70/30 liquid, 55/45 liquid, 50/50 liquid, 25/75 liquid and PDMS500 on a logarithmic scale. The solid lines correspond to the best linear fits. For clarity, the contact angle dynamics of 65/35 and 60/40 liquids are not shown.

Table 1

Slopes of the different contact angle dynamics.

\begin{tabular}{lll}
\hline Samples & Slope & Adj. R-Square \\
\hline PDMS5 & $-1.06 \pm 0.11$ & 0.993 \\
75/25 liquid & $-0.94 \pm 0.08$ & 0.985 \\
70/30 liquid & $-0.51 \pm 0.03$ & 0.997 \\
65/35 liquid & $-0.50 \pm 0.06$ & 0.995 \\
60/40 liquid & $-0.49 \pm 0.06$ & 0.997 \\
$55 / 45$ liquid & $-0.49 \pm 0.04$ & 0.995 \\
50/50 liquid & $-0.48 \pm 0.06$ & 0.994 \\
25/75 liquid & $-0.49 \pm 0.07$ & 0.995 \\
PDMS500 & $-0.52 \pm 0.04$ & 0.997 \\
\hline
\end{tabular}

or equivalently

$\ln y_{t}=\frac{1}{2} \ln t+\ln \frac{\alpha \gamma^{1 / 4} r_{0}^{1 / 4}}{\rho^{1 / 4}}$

where $\alpha$ is an adjustable parameter. Fig. 6 shows the logarithms of the meniscus height for PDMS5 and 75/25 liquid. It indicates that the early stages of the meniscus rise follow Eq. (6) and thus dominated by inertia.

After the inertial rise, the contact angles become small enough that Eq. (4) is valid for PDMS5 and 75/25 liquid, as confirmed by Fig. 5. Provided that the frictional dissipation at the triple line is dominant, the MKT model can fully describe the spreading dynamics. Therefore, the contact line velocity in Eq. (1) can be written in the linear MKT form [14]

$V=\frac{d y_{t}}{d t}=\frac{\gamma}{\zeta}\left(\cos \theta_{0}-\cos \theta_{t}\right)$

For small values of $\theta_{t}$ and $\theta_{0}=0^{\circ}$, it leads to the scaling law

$\frac{d y_{t}}{d t} \approx \frac{\gamma}{2 \zeta} \theta_{t}^{2}$

According to Eq. (4), it yields

$\frac{d y_{t}}{d t} \propto t^{-2}$

thus

$y_{t}=y_{\text {equilibrium }}-\beta_{1} t^{-1}$

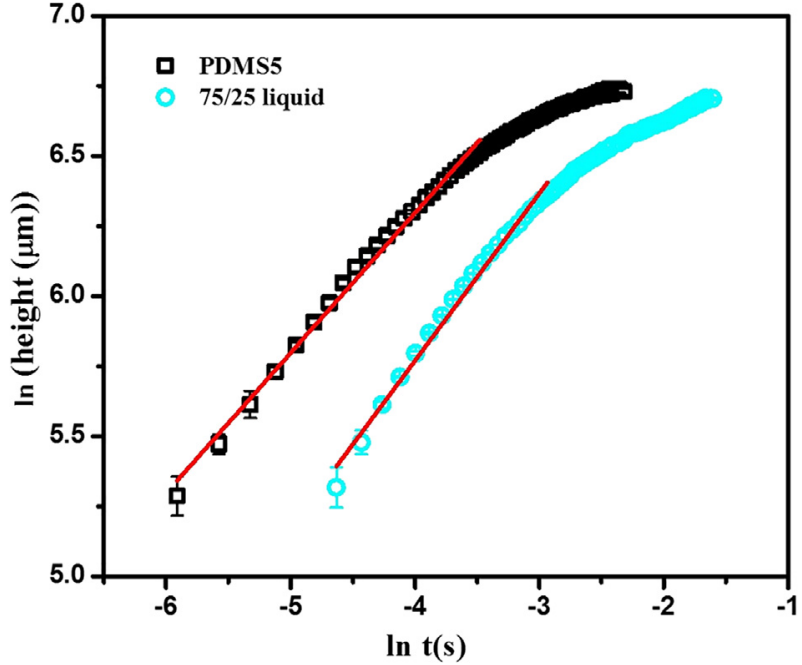

Fig. 6. Meniscus height dynamics vs time for PDMS5 and $75 / 25$ liquid on a logarithmic scale. The inertial contribution is modelled by Eq. (6). The solid lines correspond to the best linear fits with a slope of $0.499\left(R^{2}=0.993\right)$ and $0.557\left(R^{2}=\right.$ 0.985 ) for PDMS5 and 75/25 liquid, respectively.

where $y_{\text {equilibrium }}$ is the liquid height at equilibrium and $\beta_{1}$ is a numerical factor. It suggests that, after an inertial regime, the liquid rises to the equilibrium height with a $t^{-1}$ scaling law. Fig. 7 shows very good agreements between the numerical fit of Eq. (10) and the height dynamics for the PDMS5 and 75/25 liquids.

Similarly, Fig. 5 suggests that the contact angle relaxations of other liquids can be described by Eq. (3). It means that the viscous dissipation is dominant for those liquids, and the HD model can also describe the dynamics of meniscus heights. The contact line velocity in Eq. (1) can be written in this form

$V=\frac{d y_{t}}{d t}=\frac{\gamma \theta_{t}\left(\cos \theta_{0}-\cos \theta_{t}\right)}{6 \eta \ln \left(\frac{x_{\max }}{x_{\min }}\right)}$

For small values of $\theta_{t}$ and $\theta_{0}=0^{\circ}$, it leads to the scaling law

$\frac{d y_{t}}{d t} \approx \frac{\gamma}{12 \eta \ln \left(\frac{x_{\max }}{x_{\min }}\right)} \cdot \theta_{t}^{3}$

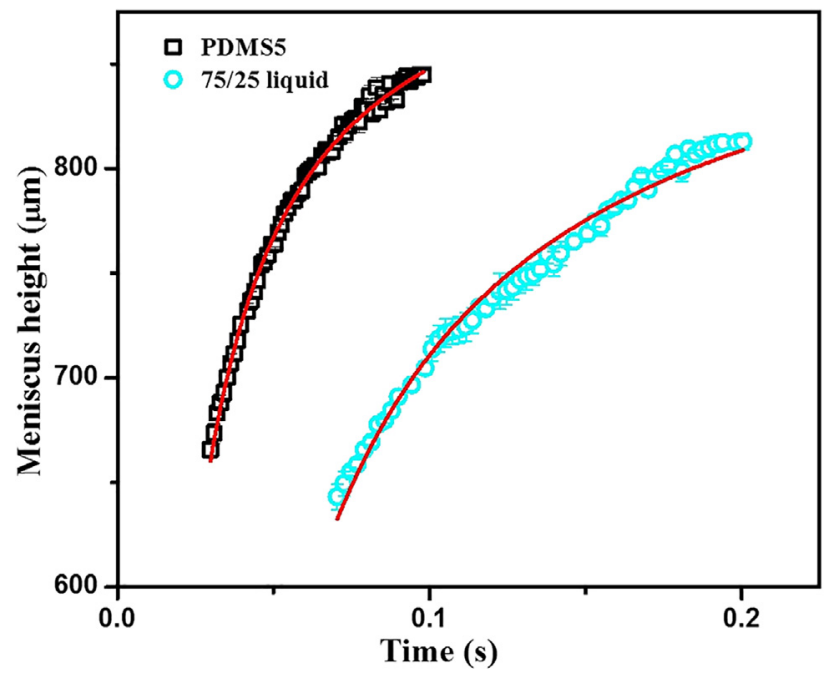

Fig. 7. Meniscus height dynamics vs time for PDMS5 and 75/25 liquid after inertial regime. The solid lines correspond to the best fit of $y_{t}=A-B t^{n}(A, B$ and $n$ are adjustable parameters) with $n=-0.963\left(R^{2}=0.997\right)$ for PDMS5 and $n=-0.918\left(R^{2}\right.$ $=0.983$ ) for $75 / 25$ liquid. 


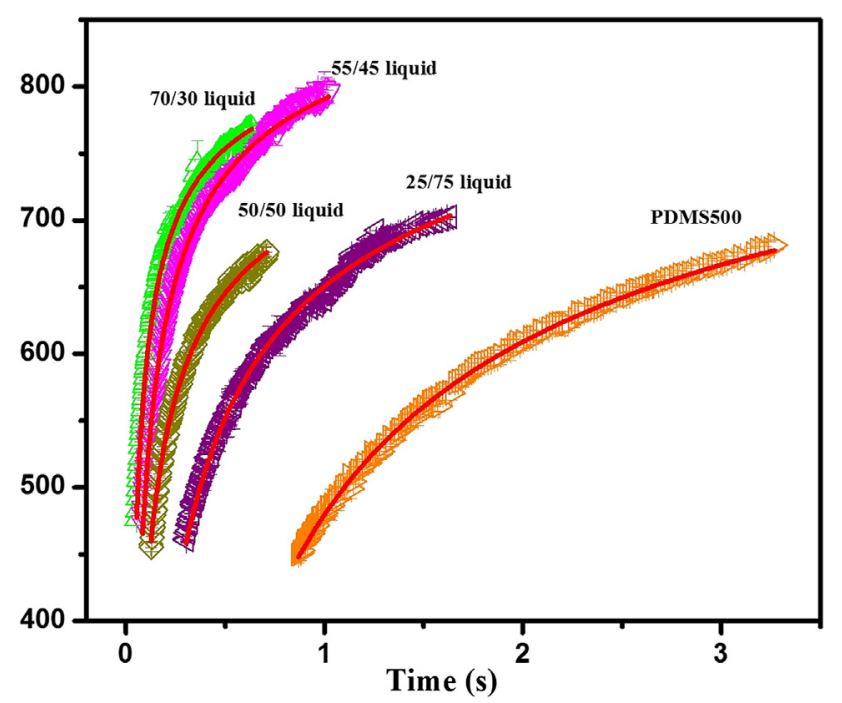

Fig. 8. Meniscus height dynamics vs time for $70 / 30$ liquid, $55 / 45$ liquid, $50 / 50$ liquid, 25/75 liquid and PDMS500 (inset) with $\theta_{t} \leq 45^{\circ}$. The solid lines correspond to the best fit of $y_{t}=A-B t^{n}$ with $n=-0.515\left(R^{2}=0.998\right)$ for $70 / 30$ liquid, $n=-0.466$ $\left(R^{2}=0.995\right)$ for $55 / 45$ liquid, $n=-0.473\left(R^{2}=0.998\right)$ for 50/50 liquid, $n=-0.461\left(R^{2}\right.$ $=0.997)$ for $25 / 75$ liquid and $n=-0.491\left(R^{2}=0.999\right)$ for PDMS500.

According to Eq. (3), it yields

$\frac{d y_{t}}{d t} \propto t^{-3 / 2}$

Thus

$y_{t}=y_{\text {equilibrium }}-\beta_{2} t^{-1 / 2}$

where $\beta_{2}$ is a numerical factor. It implies the liquid rise to the equilibrium height with a $t^{-1 / 2}$ scaling law. Fig. 8 shows that the fitting procedures lead to excellent agreements between Eq. (14) and the rise of height dynamics, indicating the HD model is relevant here.

\subsection{Comparison of the two-regimes transition between pure and mixture PDMS}

The contact angles relaxations versus time for the 75/25 liquid and for a pure PDMS liquid of viscosity $20 \mathrm{~mm}^{2} / \mathrm{s}$ (PDMS20) [22] are compared in Fig. 9. As can be seen, the corresponding slopes

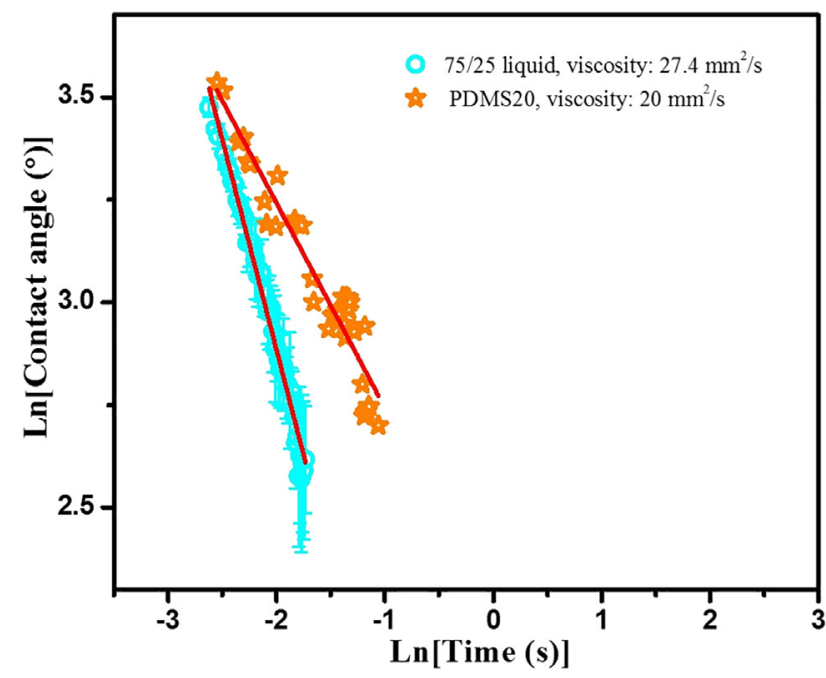

Fig. 9. Comparison between 75/25 liquid and PDMS20 in late stage contact angle dynamics. The data of PDMS20 are from Vega et al. [22] and its slope of PDMS20 is around -0.5 (Adj. R-Square $=0.964$ ).

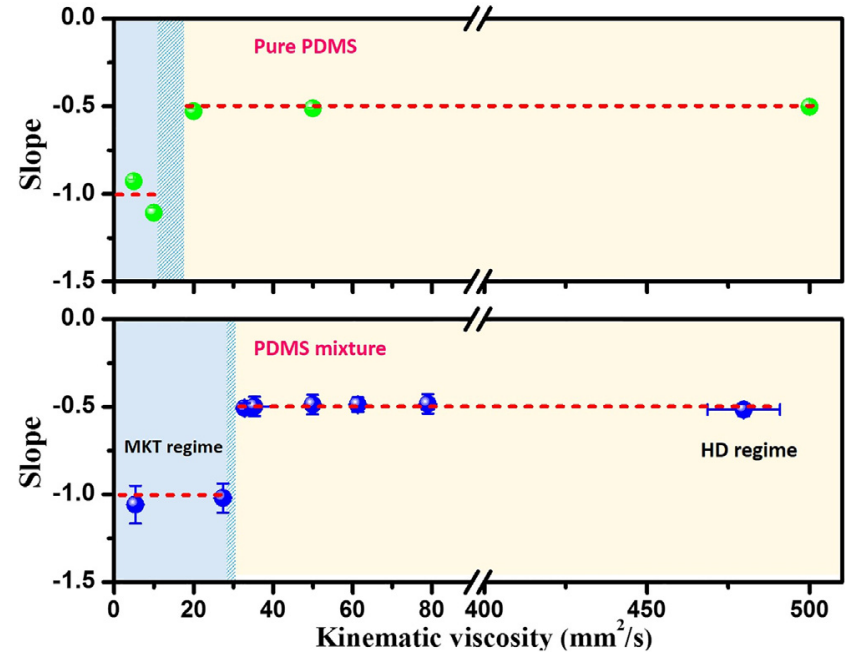

Fig. 10. Slopes of $\ln \theta$ versus $\ln t$ in one- and two-component PDMS liquids. The upper diagram (pure PDMS liquids) is adapted from Vega et al. [22].

of these two dynamics are different even though both liquids have nearly the same surface tension (Fig. 1) and equilibrium contact angle (Fig. S1). Interestingly, the 75/25 liquid with a higher viscosity follows MKT whereas the counterpart with a lower viscosity obeys HD model. For a comprehensive comparison, Fig. 10 shows a two-step function that is the slopes of $\ln \theta$ versus $\ln t$ versus the viscosities of the pure PDMS liquids [22] and the PDMS mixtures. In the work of Vega et al. [22], it is observed that for pure PDMS liquids with low viscosities, the dynamics are compatible with the MKT. For high-viscosity values, the HD model better models the dynamics. The transition between the MKT and HD regimes is observed for a viscosity between 10 and $20 \mathrm{~mm}^{2} / \mathrm{s}$ (Fig. 10). Mixing low molecular weight PDMS with high molecular weight polymeric liquids, it can be observed that this transition moves to a regime with higher viscosity, around $27.4 \sim 32.8 \mathrm{~mm}^{2} / \mathrm{s}$ (Fig. 10). Apparently, this transition range has become much narrower which may benefit from the easy-controlled viscosity variation of the mixed PDMS liquids.

The new properties of MKT/HD transition and its shift for PDMS mixtures are very intriguing. Two plausible mechanisms are proposed to account for them. When a homogenous polymer mixture having different mobile components is exposed to air, it has been confirmed that the smaller molecule component migrates to the free surface [38-41], if the concentration of big molecules is not too high. When it is too high, the surface segregation can be inhibited [39] or within the time scale required for our spreading experiments will be partially realized. This surface migration has been confirmed experimentally using X-ray photoemission spectroscopy [40] and neutron reflectivity [41]. Since the molecular size of PDMS5 is much smaller than the one of PDMS500, we do expect such segregation at the liquid/air interface. It should be noted that surface segregation can be relevant here despite the short capillary rise timescale. Indeed, previous studies have shown that time dependent surface segregation can be characterized by a non-zero intercept when the evolution of the thickness of a surface enrichment layer is extrapolated to the initial time, indicating rapid changes of the surface composition profiles in very short time $[42,43]$. For example, Wang et al. [44] took advantage of the low mobility of polymer chain segments around the glass transition temperature $\left(T_{g}\right)$ and the high depth resolution of neutron reflectivity to investigate the early stage surface segregation of polymer blends of poly(methyl methacrylate) (PMMA, $T_{g}=120$ ${ }^{\circ} \mathrm{C}$ ) and poly(styrene-ran-acrylonitrile) (SAN, $T_{g}=114{ }^{\circ} \mathrm{C}$ ) around $130^{\circ} \mathrm{C}$. They found that the process of PMMA segregation is very 


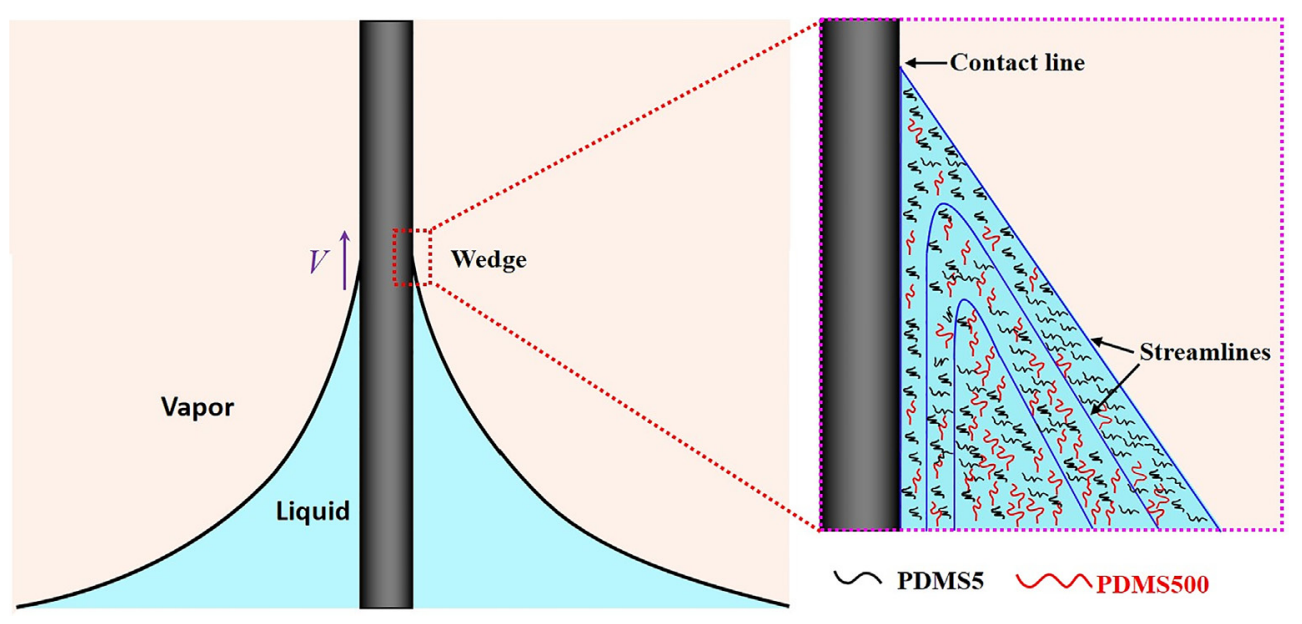

Fig. 11. Schematic illustrations of streamlines at the wedge in the vicinity of the contact line for a two-component PDMS liquid.

fast and also obtained a non-zero intercept of surface excess for PMMA by extrapolation towards zero time. Compared the mobility of PMMA with PDMS in the present study, the mobility of PDMS chain should be even higher since the test temperature $\left(20^{\circ} \mathrm{C}\right)$ is much higher than the $T_{g}$ (around $-127.5^{\circ} \mathrm{C}$ ) of PDMS [33]. Moreover, when spontaneous spreading is considered, it is known that most of the flow of molecules will be concentrated inside a ribbon just below the air-liquid interface $[1,8]$. The underlying mechanism controlling the influence of the surface segregation on the wetting regime is schematically shown in Fig. 11. More PDMS5 chains with smaller sizes tend to concentrate in the outer ribbon (region between two adjacent streamlines) due to entropy [38], as illustrated in Fig. 11. This surface segregation leads to a lower local viscosity in the vicinity of the liquid/air interface than in the bulk and this eventually determines the dominant channel of energy dissipation [45]. Therefore, the MKT/HD transition moves to a relatively high apparent viscosity regime in Fig. 10. For the same reason and despite that the viscosity of the 75/25 liquid is higher than the one of PDMS20, the spreading dynamics of the former liquid can be described by the MKT as shown in Fig. 9. However, it is difficult to experimentally quantify the local viscosity in the vicinity of the contact line because this region is supposed to be only a few nanometer thick [46]. At the nanoscale, molecular dynamics simulations may offer more detailed information on the surface segregation of short chains. It will be the purpose of a forthcoming work.

The other plausible mechanism has been suggested by Blake [47] and is related to the likely existence of a precursor film during complete wetting of silicon wafers and glasses by PDMS [5]. If this was present, there would be competition between the velocity of the contact line $V$ and the velocity of the precursor film $V_{f}$. When a precursor film exists, there is still very strong viscous dissipation [1], but the true contact line at the leading edge of the film plays no part in the creation of the apparent contact angle that is measured. Hence the conditions for the MKT are not realized. The transition would then appear naturally when $V=V_{f}$, which would explain the observed sharp character of the transition. Whenever $V>V_{f}$, a MKT regime might be observed and if $V<V_{f}$, we would obtain the HD regime. To facilitate this, all that would be required is the presence of a low viscosity component in the high viscosity PDMS. However, the fact that the transition moves to higher viscosities for the deliberate mixtures seems to argue against this hypothesis.

\section{Conclusions}

The dynamics of the rise of two-component PDMS liquids on a PET fiber is studied. The results confirmed that MKT and HD regimes exist during the spontaneous rise phenomenon. After a short period dominated by inertia, the frictional dissipation drives the low-viscosity liquid dynamics whereas the high-viscosity liquid dynamics are dominated by viscous dissipation. Compared to the rise of pure PDMS liquids, the transition between the two models moves to a higher viscosity regime for the 2-component liquids, even though the surface tension and equilibrium contact angles are nearly the same for the considered PDMS liquids. Surface segregation of shorter molecules or precursor film may be responsible for this displaced transition. Our results suggest that a new transition can take place readily from a polymer mixture having components of different molecular weights. This finding has interesting implications in several practical applications, which are related to chocolate preparation, hot-melt adhesives, food packaging $[39,41,48]$. It also helps to explain why some very viscous industrial polymers [49] can be described by the MKT.

According to the present work, it is still difficult to make rigorous mathematical claims as to why there is a sharp transition between MKT and HD models since the theoretical basis of the two models mostly differs from each other. It is also difficult to offer experimental evidence of surface segregation for the shift of MKT/HD transition because the contact line region is at the nanoscale and the system lies in a dynamic process. An alternative methodology is large scale molecular dynamics simulations from which the underlying mechanisms driving the spreading dynamics should be unraveled. This will be explored in future works.

\section{Acknowledgments}

The authors gratefully acknowledge Dr. T.D. Blake for fruitful discussions and critical comments on this manuscript. Thanks are also given to Dr. J.C. Fernandez-Toledano and Mr. M. Amin Ali for their help in the analysis of the experimental data. This research has been partially funded by the Interuniversity Attraction Poles Programme (IAP 7/38 MicroMAST) initiated by the Belgian Science Policy Office.

\section{Appendix A. Supplementary data}

Supplementary data associated with this article can be found, in the online version, at https://doi.org/10.1016/j.jcis.2018.04.091.

\section{References}

[1] P.G. de Gennes, Wetting: statics and dynamics, Rev. Mod. Phys. 57 (3) (1985) $827-863$. 
[2] D. Bonn, J. Eggers, J. Indekeu, J. Meunier, E. Rolley, Wetting and spreading, Rev. Mod. Phys. 81 (2) (2009) 739-805.

[3] J. De Coninck, T.D. Blake, Wetting and molecular dynamics simulations of simple liquids, Annu. Rev. Mater. Res. 38 (2008) 1-22.

[4] F. Brochard, Spreading of liquid drops on thin cylinders: the "manchon/droplet" transition, J. Chem. Phys. 84 (8) (1986) 4664-4672.

[5] P.G. de Gennes, F. Brochard-Wyart, D. Quéré, Capillarity and Wetting Phenomena: Drops, Bubbles, Pearls, Waves, Springer-Verlag, New York, 2004.

[6] T.H. Chou, S.J. Hong, Y.E. Liang, H.K. Tsao, Y.J. Sheng, Equilibrium phase diagram of drop-on-fiber: coexistent states and gravity effect, Langmuir 27 (7) (2011) 3685-3692.

[7] S. Lim, H. Zhang, P. Wu, A. Nikolov, D. Wasan, The dynamic spreading of nanofluids on solid surfaces-role of the nanofilm structural disjoining pressure, J. Colloid Interface Sci. 470 (2016) 22-30.

[8] C. Huh, L. Scriven, Hydrodynamic model of steady movement of a solid/liquid/ fluid contact line, J. Colloid Interface Sci. 35 (1) (1971) 85-101.

[9] O. Voinov, Hydrodynamics of wetting, Fluid Dyn. 11 (5) (1976) 714-721.

[10] L. Tanner, The spreading of silicone oil drops on horizontal surfaces, J. Phys. D: Appl. Phys. 12 (9) (1979) 1473.

[11] R. Cox, The dynamics of the spreading of liquids on a solid surface. Part 1. Viscous flow, J. Fluid Mech. 168 (1986) 169-194.

[12] T.D. Blake, J.M. Haynes, Kinetics of liquid/liquid displacement, J. Colloid Interface Sci. 30 (3) (1969) 421-423.

[13] T.D. Blake, Dynamic Contact Angles and Wetting Kinetics, in: J.C. Berg (Ed.), Wettability, Marcel Dekker, New York, 1993, pp. 251-309.

[14] T.D. Blake, The physics of moving wetting lines, J. Colloid Interface Sci. 299 (1) (2006) 1-13.

[15] A.M. Karim, J.P. Rothstein, H.P. Kavehpour, Experimental study of dynamic contact angles on rough hydrophobic surfaces, J. Colloid Interface Sci. 513 (2018) 658-665.

[16] R. Sedev, The molecular-kinetic approach to wetting dynamics: achievements and limitations, Adv. Colloid Interface Sci. 222 (2015) 661-669.

[17] D. Ausserré, A. Picard, L. Léger, Existence and role of the precursor film in the spreading of polymer liquids, Phys. Rev. Lett. 57 (21) (1986) 2671.

[18] M.J. de Ruijter, M. Charlot, M. Voué, J. De Coninck, Experimental evidence of several time scales in drop spreading, Langmuir 16 (5) (2000) 2363-2368.

[19] D. Quéré, J.-M. Di Meglio, The meniscus on a fibre, Adv. Colloid Interface Sci. 48 (1994) 141-150.

[20] C. Clanet, D. Quéré, Onset of menisci, J. Fluid Mech. 460 (2002) 131-149.

[21] D. Seveno, J. De Coninck, Possibility of different time scales in the capillary rise around a fiber, Langmuir 20 (3) (2004) 737-742.

[22] M.J. Vega, D. Seveno, G. Lemaur, M.H. Adão, J. De Coninck, Dynamics of the rise around a fiber: experimental evidence of the existence of several time scales, Langmuir 21 (21) (2005) 9584-9590.

[23] Y.C. Zhang, D. Zheng, H. Pang, J.H. Tang, Z.M. Li, The effect of molecular chain polarity on electric field-induced aligned conductive carbon nanotube network formation in polymer melt, Compos. Sci. Technol. 72 (15) (2012) 1875-1881.

[24] A.R. Harikrishnan, P. Dhar, S. Gedupudi, S.K. Das, Effect of interaction of nanoparticles and surfactants on the spreading dynamics of sessile droplets, Langmuir 33 (43) (2017) 12180-12192.

[25] L.N. Nwidee, M. Lebedev, A. Barifcani, M. Sarmadivaleh, S. Iglauer, Wettability alteration of oil-wet limestone using surfactant-nanoparticle formulation, J. Colloid Interface Sci. 504 (2017) 334-345.
[26] U. Steiner, J. Klein, E. Eiser, A. Budkowski, L.J. Fetters, Complete wetting from polymer mixtures, Science 258 (5085) (1992). 1126-1126.

[27] D.R. Heine, G.S. Grest, E.B. Webb III, Spreading dynamics of polymer nanodroplets in cylindrical geometries, Phys. Rev. E 70 (1) (2004) 011606.

[28] C.F. Brooks, A.M. Grillet, J.A. Emerson, Experimental investigation of the spontaneous wetting of polymers and polymer blends, Langmuir 22 (24) (2006) 9928-9941.

[29] D. Seveno, T.D. Blake, S. Goossens, J. De Coninck, Predicting the wetting dynamics of a two-liquid system, Langmuir 27 (24) (2011) 14958-14967.

[30] D. Duvivier, D. Seveno, R. Rioboo, T.D. Blake, J. De Coninck, Experimental evidence of the role of viscosity in the molecular kinetic theory of dynamic wetting, Langmuir 27 (21) (2011) 13015-13021.

[31] A.C.M. Kuo, Poly(dimethylsiloxane), in: J.E. Mark (Ed.), Polymer Data Handbook, Oxford University Press, Oxford, 1999, pp. 411-435.

[32] M. Roth, Solubility parameter of poly(dimethyl siloxane) as a function of temperature and chain length, J. Polym. Sci. Part B: Polym. Phys. 28 (13) (1990) $2715-2719$.

[33] J.E. Mark, Physical Properties of Polymers Handbook, second ed., Springer, New York, 2007.

[34] R. Fondecave, F. Brochard-Wyart, Wetting laws for polymer solutions, Europhys. Lett. 37 (2) (1997) 115.

[35] T. Cosgrove, M. Turner, P. Griffiths, J. Hollingshurst, M. Shenton, J. Semlyen, Self-diffusion and spin-spin relaxation in blends of linear and cyclic polydimethylsiloxane melts, Polymer 37 (9) (1996) 1535-1540.

[36] M.J. de Ruijter, J. De Coninck, G. Oshanin, Droplet spreading: partial wetting regime revisited, Langmuir 15 (6) (1999) 2209-2216.

[37] P.G. de Gennes, Deposition of langmuir-blodgett layers, Colloid Polym. Sci. 264 (5) (1986) 463-465.

[38] A. Hariharan, S.K. Kumar, T.P. Russell, Surface segregation in binary polymer mixtures: a lattice model, Macromolecules 24 (17) (1991) 4909-4917.

[39] J. Krawczyk, S. Croce, T. McLeish, B. Chakrabarti, Elasticity dominated surface segregation of small molecules in polymer mixtures, Phys. Rev. Lett. 116 (20) (2016) 208301.

[40] D.H.K. Pan, W. Prest Jr, Surfaces of polymer blends: X-ray photoelectron spectroscopy studies of polystyrene/poly (vinyl methyl ether) blends, J. Appl. Phys. 58 (8) (1985) 2861-2870.

[41] E.F. Sabattié, J. Tasche, M.R. Wilson, M.W. Skoda, A. Hughes, T. Lindner, R.L. Thompson, Predicting oligomer/polymer compatibility and the impact on nanoscale segregation in thin films, Soft Matter 13 (19) (2017) 3580-3591.

[42] G. Krausch, C.-A. Dai, E.J. Kramer, F.S. Bates, Real space observation of dynamic scaling in a critical polymer mixture, Phys. Rev. Lett. 71 (22) (1993) 3669.

[43] U. Steiner, J. Klein, Growth of wetting layers from liquid mixtures, Phys. Rev. Lett. 77 (12) (1996) 2526.

[44] H. Wang, J.F. Douglas, S.K. Satija, R.J. Composto, C. Han, Early-stage compositional segregation in polymer-blend films, Phys. Rev. E 67 (6) (2003) 061801.

[45] C.W. Extrand, Origins of wetting, Langmuir 32 (31) (2016) 7697-7706.

[46] T.D. Blake, J.C. Fernandez-Toledano, G. Doyen, J. De Coninck, Forced wetting and hydrodynamic assist, Phys. Fluids 27 (11) (2015) 112101.

[47] T.D. Blake, Personal Communication, Université de Mons, 2018.

[48] P. Lonchampt, R.W. Hartel, Fat bloom in chocolate and compound coatings Eur. J. Lipid Sci. Technol. 106 (4) (2004) 241-274.

[49] Y. Zhang, C.A. Fuentes, R. Koekoekx, C. Clasen, A.W. Van Vuure, J. De Coninck, D. Seveno, Spreading dynamics of molten polymer drops on glass substrates, Langmuir 33 (34) (2017) 8447-8454. 